\title{
A study on UV emission from clouds with Mini-EUSO
}

\author{
Alessio Golzio, ${ }^{a, b}, *$ Matteo Battisti, ${ }^{a, b}$ Mario Bertaina, ${ }^{a}$ Karl Bolmgren, ${ }^{c}$ Giorgio \\ Cambiè, ${ }^{d}$ Marco Casolino, ${ }^{d, e, i}$ Claudio Cassardo, ${ }^{b}$ Roberto Cremonini, ${ }^{f}$ Silvia \\ Ferrarese,${ }^{b}$ Christer Fuglesang, ${ }^{c}$ Massimiliano Manfrin, ${ }^{b}$ Laura Marcelli, ${ }^{e}$ Lech \\ Piotrowski $^{g}$ and Kenji Shinozaki ${ }^{h}$ on behalf of the JEM-EUSO Collaboration
} (a complete list of authors can be found at the end of the proceedings)

${ }^{a}$ INFN, Sezione di Torino - Torino, Italy

${ }^{b}$ Universitá di Torino, Department of Physics - Torino, Italy

${ }^{c}$ KTH Royal Institute of Technology, Department of Physics - Stockholm, Sweden

${ }^{d}$ Universitá degli Studi di Roma Tor Vergata - Dipartimento di Fisica, Roma, Italy

${ }^{e}$ INFN, Sezione di Roma Tor Vergata - Roma, Italy

${ }^{f}$ ARPA Piemonte - Torino, Italy

${ }^{g}$ Faculty of Physics, University of Warsaw - Warsaw, Poland

${ }^{h}$ National Centre for Nuclear Research - Lodz, Poland

${ }^{i}$ RIKEN Wako - Japan

E-mail: alessio.golzio@unito.it

Mini-EUSO is the first mission of the JEM-EUSO program located on the International Space Station. One of the main goals of the mission is to provide valuable scientific data in view of future large missions devoted to study Ultra-High Energy Cosmic Rays (UHECRs) from space by exploiting the fluorescence emission generated by Extensive Air Showers (EAS) developing in the atmosphere. A space mission like Mini-EUSO experiences continuous changes in atmospheric conditions, including the cloud presence. The influence of clouds on space-based observation is, therefore, an important topic to investigate as it might alter the instantaneous exposure for EAS detection or deteriorate the quality of the EAS images with consequences on the reconstructed EAS parameters. For this purpose, JEM-EUSO is planning to have an IR camera and a lidar as part of its Atmospheric Monitoring System. At the same time, it would be extremely beneficial if the UV camera itself would be able to detect the presence of clouds, at least in some specific conditions. For this reason, we analyze a few case studies by comparing the pixel count rates from Mini-EUSO during orbits with the cloud cover (as cloud fraction). This quantity is retrieved from the Global Forecast System (GFS) model at different height levels over the Mini-EUSO trajectory. The results of this analysis are reported.

$37^{\text {th }}$ International Cosmic Ray Conference (ICRC 2021)

- July 12th - 23rd, 2021

Online - Berlin, Germany

\footnotetext{
*Presenter
} 


\section{Introduction}

The cloud cover determination is an open issue, and affects several experimental fields, from the physics of the atmosphere (meteorology and climatology), to astronomy, and to cosmic-ray detection system. The Mini-EUSO telescope observes the Earth in the ultraviolet band (UV) [1], and is devoted to the study of ultra high energy cosmic rays coming from the space through their extensive air showers in the Earth's atmosphere. From a first inspection of Mini-EUSO UV maps it was possible to state the capability of this telescope to detect tropospheric clouds (see Figure 1). In this particular event a tropical cyclone was detected in the Indian Ocean north-east of Madagascar Island (Figure 1a, and a zoom in Figure 1c). The spiral-form of the cloud is also visible in the RGB Dust composite Image of METEOSAT8, the meteorological geostationary satellite, reported in Figure 1b.

The Mini-EUSO telescope is on board of International Space Station (ISS) that completes an orbit around the Earth in $\approx 90$ minutes. The covered area is a $350-\mathrm{km}$ wide line oscillating between $50^{\circ}$ North-South. The size of the considered area makes the cloud data retrieval from satellites very complex due to the fragmentation of the archives and the availability of images. So we decided, as a first step, to compare the UV maps with the cloud cover predicted by a Numeric Weather Prediction model (NWP) of the United States' National Weather Service, the Global Forecast System (GFS), that is available $24 \mathrm{~h}-7$ days at a resolution of $0.25^{\circ}$ and with a 1-hour forecast step.

The actual NWPs compute the cloud fraction at each model level from pressure, cloud condensation mixing ratio, relative humidity and temperature data [2]. NWPs predict the place (grid point), the amount and the thickness of the cloud on a discrete vertical and horizontal grid. The accuracy of NWPs is in general high (more than 70\%), with a forecast error less than $30 \%$ in the first hours [3]. The cloud mask, resulting in a 2D, latitude-longitude, true/false fields, may be computed at high horizontal resolution using the NWPs cloud fraction data, following the same methodology applied to evaluate the cloud top height in [4]. In the present work, in order to obtain more accurate results, different thresholds are applied for high, medium and low clouds, and the cloud mask is compared with the UV maps from Mini-EUSO, to detect the cloud cover from UV data, a new application of this dataset. The results are presented and discussed.

\section{Data and analysis}

Even though 41 Mini-EUSO sessions have been registered up to now, in this paper only 14 sessions are used, considering the availability of GFS high-resolution data (available only when the session was taken) and the UV maps. The available data for each of these sessions correspond to $\approx 44$ minutes, coming from the first and/or the last part of the acquisition. The analysis is conducted both on all Mini-EUSO available trajectories (over land and sea regions) and on the sea regions only, to avoid the contamination coming from antropogenic light. The main light-source in space is due to the reflectance of the Moon (its phase is reported in Figure 3 as percentage of full-Moon) and the nightglow/airglow signals, while the sources from the Earth are the cloud reflection (of space light), lightnings (not visible in the data because they were triggered by the sensor), boat lights or other atmospheric events. The session considered span from 15 September 2020 to 6 May 2021. 


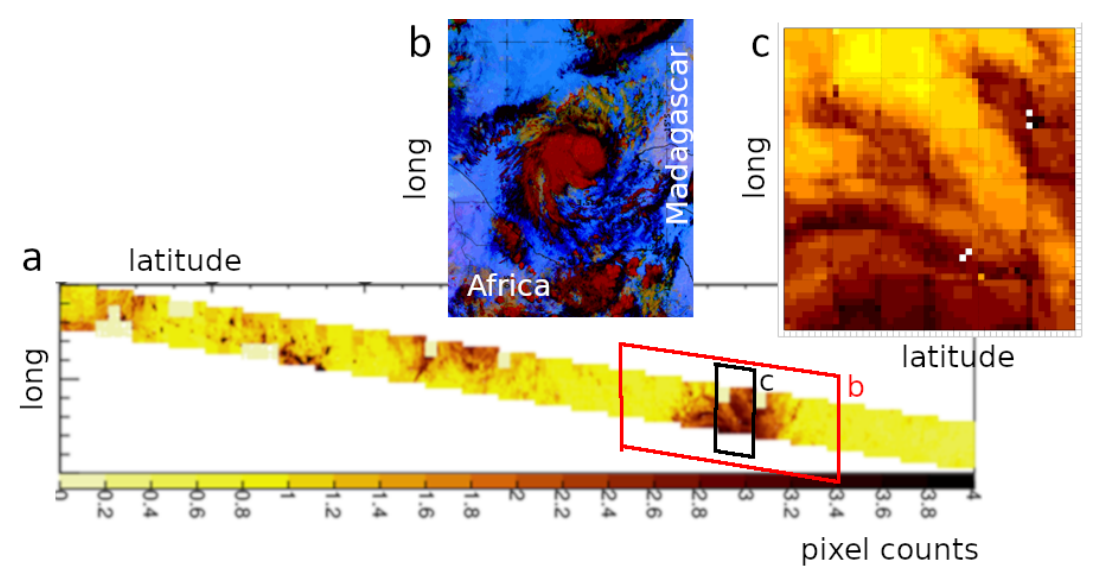

Figure 1: a) composite image of the Mini-EUSO pixel counts over the Indian Ocean at approximately 18.38 UTC of December 5, 2019; b) a RGB Dust Composite image from the METEOSAT 8 meteorological satellite, showing the tropical cyclone seen by Mini-EUSO; c) a single image of the Mini-EUSO pixel matrix where the banded clouds of the cyclone are clearly visible. In the first part of the image, panel a), the dotted points are middle-low cumulus clouds.

The area compared in each session over sea span from $2.61 \times 10^{6} \mathrm{~km}^{2}$ to $6.60 \times 10^{6} \mathrm{~km}^{2}$, while over sea and land span from $5.73 \times 10^{6} \mathrm{~km}^{2}$ to $7.30 \times 10^{6} \mathrm{~km}^{2}$.

\section{Methods}

\subsection{UV maps and regridding}

Before the UV observations can be used, the possibility of inter-pixel differences (e.g. differences in pixel efficiency) must be accounted for. The different pixels of the Mini-EUSO detector are therefore subjected to a relative calibration using the lowest count of each pixel during the entire session as a baseline. This is possible because the physical environment (e.g. a cloudless ocean) producing this measurement should be the same for each pixel.

The original pixel resolution of the Mini-EUSO telescope at ground is about $6.3 \mathrm{~km}$, and the matrix is composed by 48 by 48 pixels with a combined field of view of about $350 \times 350 \mathrm{~km}$. The photo-sensor registers the light in counts per pixel per time step (the acquisition is every $41 \mathrm{~ms}$ ), and to make possible the comparison between the coarser GFS resolution a regridding operation is necessary.

This was done following three steps:

1. associate the center of each matrix-pixel-time observation with the corresponding latitude and longitude coordinates. This is done by projecting the shape of the detector pixel array onto the surface of the Earth by knowing the position of the ISS and the detector geometry. These calculations are made in two polar coordinate systems, one centred at the point directly beneath the ISS and one with the centre at the focal surface of the detector. Knowing the ISS altitude, $h_{i s s}$, and the part of the field of view taken up by one pixel, $F o V_{p}$, the radial 
distance $\rho_{g}$ between an observation on the ground and the ISS subpoint can be estimated as

$$
\rho_{g}=\rho_{p} h_{i s s} \tan \left(F o V_{p}\right)
$$

where $\rho_{p}$ is the radial distance (in units of pixels) to the pixel from the center of the detector, in the detector coordinate system. With the angular coordinate $\theta_{g}$ on the ground given by the ISS orientation and the corresponding angle $\theta_{p}$ on the detector, the latitude $\varphi$ and longitude $\lambda$ of the pixels as mapped to the the Earth are estimated by $\left(R_{E}=6370 \mathrm{~km}\right.$ is the Earth radius):

$$
\begin{gathered}
\varphi=\sin ^{-1}\left(\sin \varphi_{i s s} \cdot \cos \frac{\rho_{g}}{R_{E}}+\cos \varphi_{i s s} \cdot \sin \frac{\rho_{g}}{R_{E}} \cdot \cos \theta_{g}\right) \\
\lambda=\lambda_{i s s}+\tan ^{-1}\left(\frac{\sin \theta_{g} \cdot \sin \frac{\rho_{g}}{R_{E}} \cdot \cos \varphi_{i s s}}{\cos \frac{\rho_{g}}{R_{E}}-\sin \varphi_{i s s} \cdot \sin \varphi_{i s s}}\right)
\end{gathered}
$$

2. collect overlapping pixels by making spatial averages (in $41 \mathrm{~ms}$ the ISS moves of $\approx 300 \mathrm{~m}$ )

3. regrid on a $0.25^{\circ}$ longitude-latitude grid at the same center-points of GFS, considering the average of the pixel-counts within each bin formed by the grid.

\subsection{GFS an UV cloud masks computation}

The GFS data ${ }^{1}$ used in the present work consist of the cloud fraction fields with a grid spacing of $0.25^{\circ}$ and 39 vertical levels (from $1 \times 10^{5} \mathrm{~Pa}$ to $5 \times 10^{3} \mathrm{~Pa}$ ), resulting in 40491360 points for each session.

Cloud fraction values span from 0 to 1 , where 0 is clear sky and 1 overcast sky. In order to calculate the cloud mask for low, middle and high layers, the cloud fraction maximum is identified in the three layers and its value is compared with the following thresholds: Low clouds $(z<2 \mathrm{~km}$ a.s.1.) are present if maximum low-cloud fraction overpass 0.2; Medium clouds $(2<z<6 \mathrm{~km}$ a.s.l.) are present if maximum medium-cloud fraction overpass 0.4 ; High clouds $(6<z<18 \mathrm{~km}$ a.s.l.) are present if maximum high-cloud fraction overpass 0.8 . This set of thresholds was chosen after studies [e.g. 4] that show the different ability of the global model to detect the three levels of clouds. Three cloud cover masks are computed respectively for low, medium and high layers, the total cloud mask is the union of these three masks.

The UV cloud mask is computed on the UV mean counts re-sampled at the same grid spacing of GFS, using three different thresholds: 1,5 and 10 counts per grid point.

\subsection{Contingency tables}

To assess the differences in the cloud masks a contingency table is built between each cloud level and UV threshold and for every session. The cloud mask from the global model is considered the truth, while the cloud mask from Mini-EUSO UV band is considered the test, so the contingency table is built following Figure 2.

The two considered indexes are the Accuracy (Proportion Correct, PC) and the Heidke Skill Score [HSS, 5], the first indicates the hits (true positive and true negative) with respect to the overall

\footnotetext{
${ }^{1}$ The GFS operative data are real-time retrived from https://nomads.ncep.noaa.gov/cgi-bin/filter_gfs_ 0p25_1hr.pl
} 


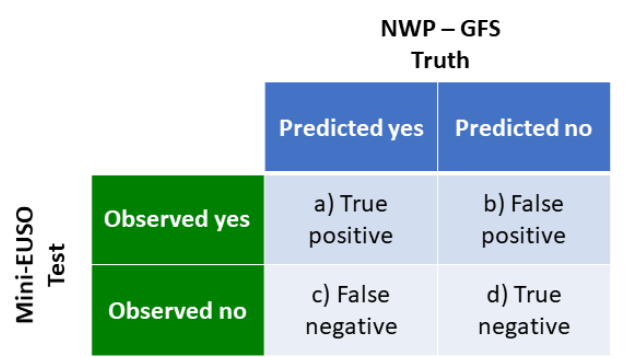

Figure 2: The contingency table used in this study. Letters refer to the calculated indexes of 3.3

cases, while the HSS indicates the quality of the forecast, and measures the fraction of correct forecasts after eliminating those forecasts which would be correct due purely to random chance.

$\mathrm{PC}$ is defined as (refer to Figure 2):

$$
P C=\frac{a+d}{a+b+c+d}=\frac{a+d}{n}
$$

The perfect value for PC is the unity (or here a percentage if PC is multiplied by 100). Its maximum-likelihood estimate is

$$
E=\left(\frac{a+c}{n}\right)\left(\frac{a+b}{n}\right)+\left(\frac{b+d}{n}\right)\left(\frac{c+d}{n}\right)
$$

HSS is defined as:

$$
H S S=\frac{P C-E}{1-E}=\frac{2(a d-b c)}{(a+b)(b+d)+(a+c)(c+d)}
$$

The HSS ranges between $-\infty$ and 1, where 1 is the perfect forecast, 0 indicates no-skill and negative values indicates that the chance forecast is better.

\section{Results and discussion}

The general statistics (Table 1) show the higher performances with the UV threshold set at one count per pixel, with slightly better results in the accuracy of low clouds over the sea (61.0\%), while over the entire dataset, middle (total) cloud cover obtain the best value $(60.8 \%)$. The HSS shows similar results regarding the UV thresholds but with a more significant gap. Again the low-clouds prediction obtained the best results: 0.12 on the sea and 0.17 over land and sea together.

\subsection{Sea-data results}

We considered only sea points to improve the analysis and remove the pixel counts related to land lights. The first question was to find a possible correlation with the Moon phase, as it is the most important light source during the night. Linear regression Pearson correlation coefficient is calculated between the accuracy and the HSS indexes and the Moon phase. The considered cases are the three cloud levels with the three UV thresholds on the two indexes (Accuracy and HSS). The correlation shows low values mainly due to the limited statistics, never higher than 0.17 , and the most correlated series with the moon phase is the low cloud HSS with the higher UV threshold $\left(R^{2}=0.166\right)$. The poor correlation values and the flatness of linear regression lines indicate that 
Table 1: Averaged results over the 14 sessions considered. Medium-cloud values represent also the Total cloud cover statistics.

\begin{tabular}{lccrrrrr}
\hline \multirow{2}{*}{ Points location } & UV threshold & \multicolumn{3}{c}{ Accuracy $(100 P C)$} & \multicolumn{4}{c}{ HSS } \\
& & High & Medium & Low & High & Medium & Low \\
\hline \multirow{3}{*}{ Sea $\left(6.48 \times 10^{7} \mathrm{~km}^{2}\right)$} & 5 & 48.3 & 59.6 & 61.1 & 0.019 & 0.072 & 0.119 \\
& 10 & 59.0 & 40.1 & 57.0 & 0.009 & 0.047 & 0.088 \\
All $\left(9.48 \times 10^{7} \mathrm{~km}^{2}\right)$ & 1 & 47.4 & 60.8 & 56.7 & 0.050 & 0.108 & 0.167 \\
& 5 & 48.8 & 42.2 & 58.3 & 0.009 & 0.052 & 0.091 \\
& 10 & 50.5 & 38.1 & 58.0 & 0.015 & 0.048 & 0.080 \\
\hline
\end{tabular}

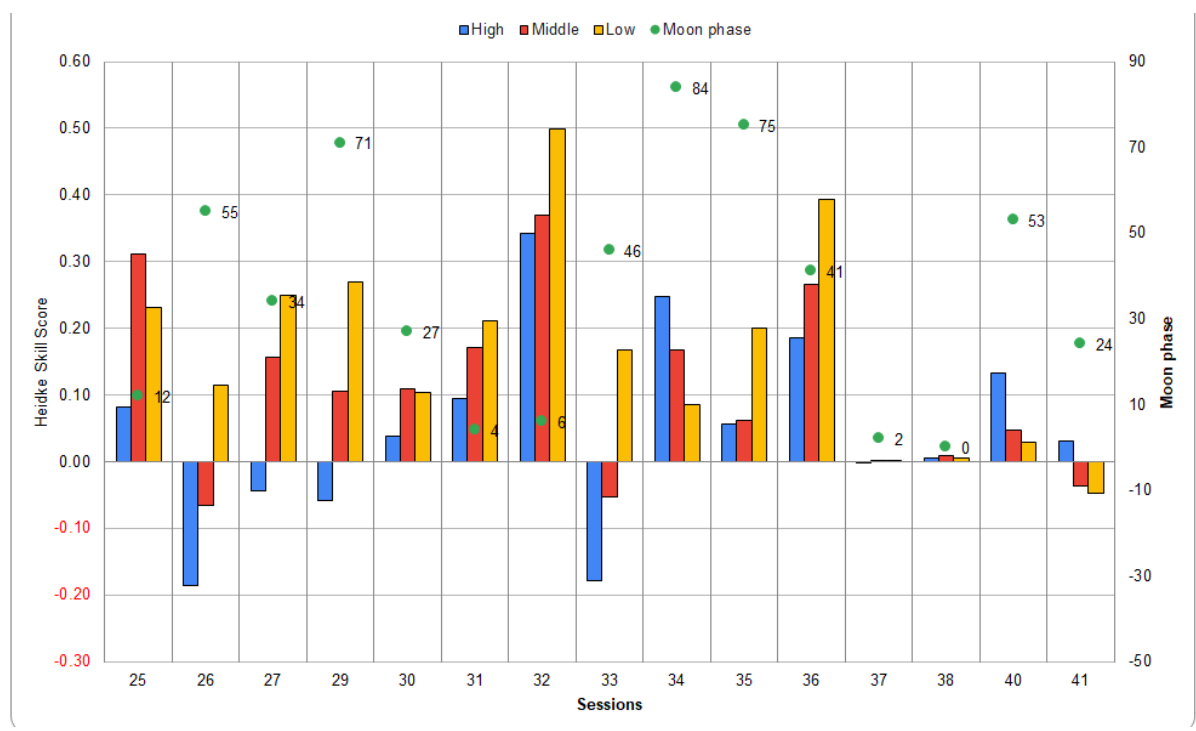

Figure 3: The histogram of HSS with the UV threshold 1 and the sea data only.

the moon does not affect the detection of clouds. Moreover, considering some single situation, the light curve from Mini-EUSO suggests that the most appropriate threshold is 1 (as also deducted from beginning of Section 4 and Table 1).

The accuracy values of UV threshold 1 are between $30 \%$ and $82 \%$, but sometimes very high accuracy values present very low (near zero) HSS, indicating a poor agreement between observation and prediction. This is the case of session 37 (Figures 3 and 4).

Analyzing the two indexes together, two sessions appear especially interesting: 32 and 36 . In these two cases, the Moon phase was respectively $6 \%$ and $41 \%$, confirming the slight influence of the quantity of moon light, and accuracy is $70.14 \%$ and $64.06 \%$, while HSS is 0.40 and 0.28 . In particular HSS has very high and promising values.

In Figure 5 the cloud masks calculated for session 36 is shown. It is possible to see that when the threshold of 1 is overpassed on pixel counts clouds are present, while in clear sky zones the UV map shows no pixel counts (white is below the threshold). 


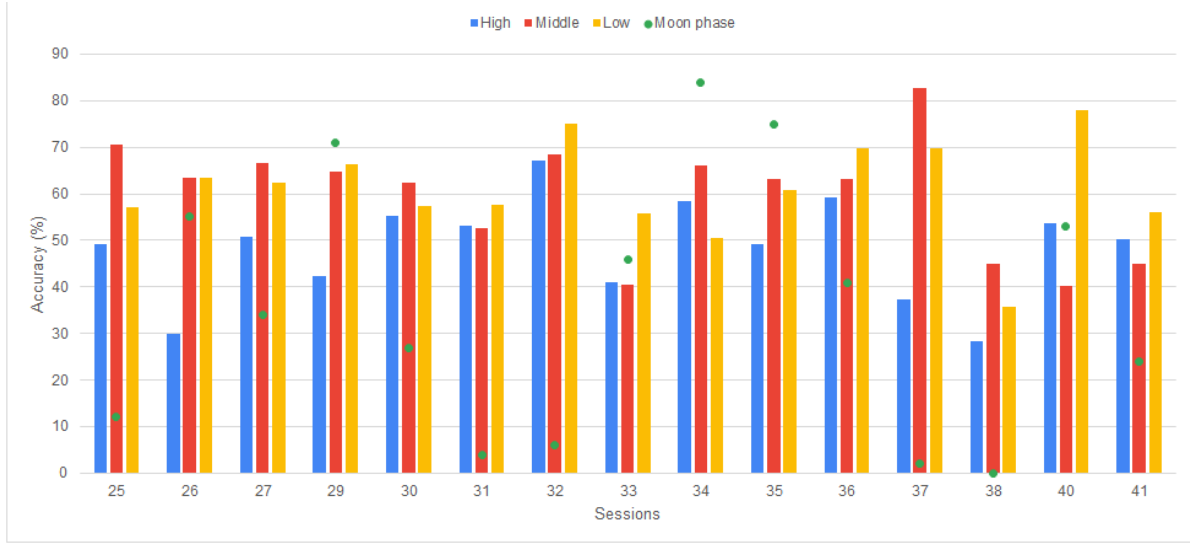

Figure 4: The histogram of Accuracy with the UV threshold 1 and the sea data only.

\section{Conclusions}

This study, considering the complexity of cloud detection and forecast, shows the ability of Mini-EUSO UV sensor to detect cloud cover during its operative time. The best UV threshold to compute the cloud mask appears to be one count per pixel, and the overall cloud detection was good (mainly in the two reported examples of session 32 and 36), also considering the degradation of the UV maps to reach the same grid spacing of GFS. From the first inspection showed in Figure 1, and from the complete statistics over the 14 session considered, it is possible to state that the Mini-EUSO sensor is able to capture the middle and low cloud better than high clouds, that usually are thinner and have a smaller optical depth. However they are able able to modify the detection of the EAS.

Future improvements and investigations will increase the reference NWP resolution and reach a greater detail in cloud cover to be compared.

\section{Acknowledgments}

This work was supported by State Space Corporation ROSCOSMOS, by the Italian Space Agency through the ASI INFN agreement n. 2020-26-HH.0 and contract n. 2016-1-U.0, by the French space agency CNES, National Science Centre in Poland grant 2017/27/B/ST9/02162. This research has been supported by the Interdisciplinary Scientific and Educational School of Moscow University "Fundamental and Applied Space Research". The article has been prepared based on research materials carried out in the space experiment "UV atmosphere".

\section{References}

[1] S. Bacholle, P. Barrillon, M. Battisti, A. Belov, M. Bertaina, F. Bisconti et al., Mini-EUSO mission to study earth UV emissions on board the ISS, The Astrophysical Journal Supplement Series 253 (2021) .

[2] K.M. Xu and D.A. Randall, A semiempirical cloudiness parameterization for use in climate models, Journal of Atmospheric Sciences 53 (1996) 3084. 

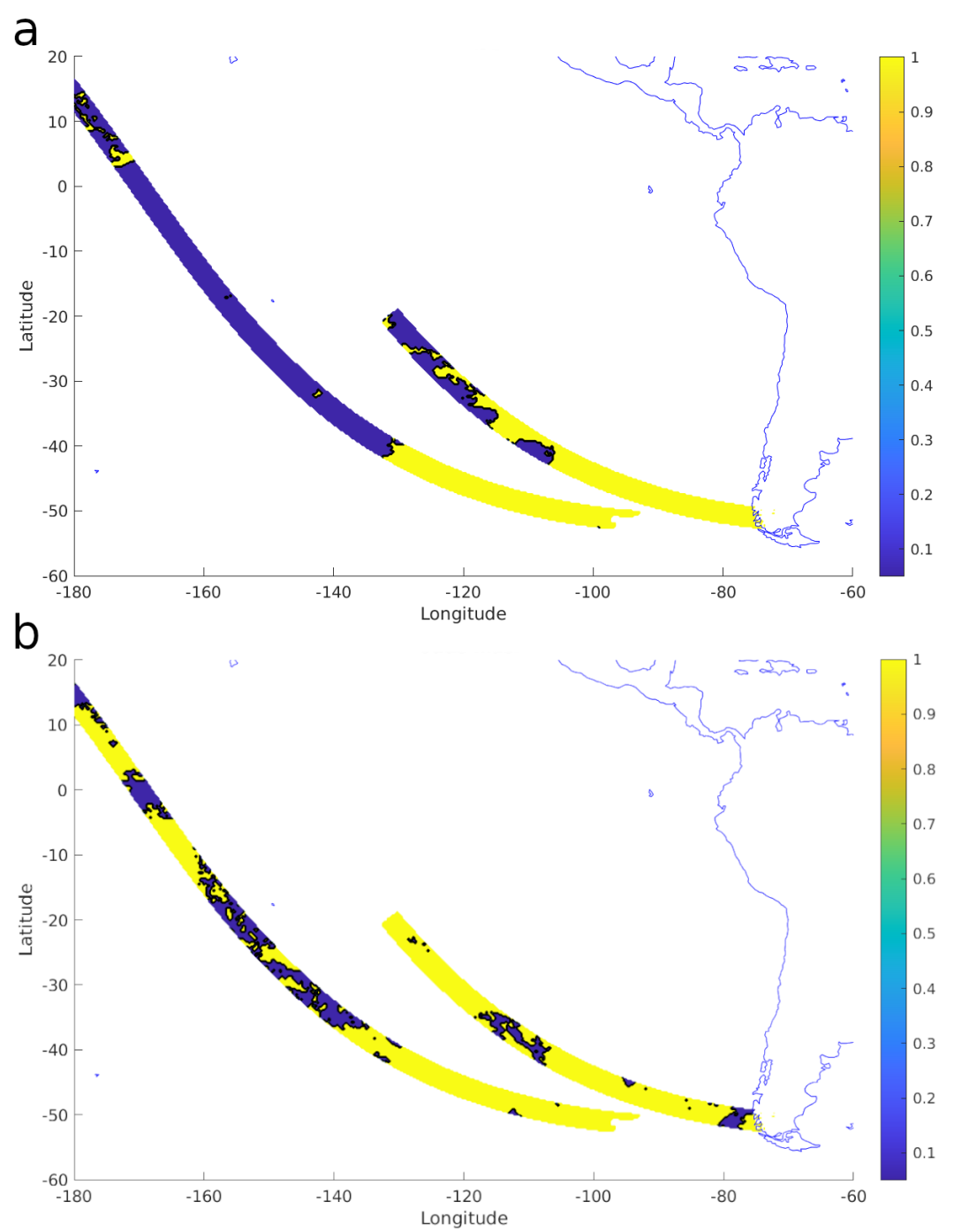

Figure 5: Session 36, 6 February 2021. a) The cloud mask computed on the UV counts from Mini-EUSO, the visible points are the pixels that pass the threshold of 1 count per pixel; b) the cloud mask from the GFS cloud fraction, as the union of low, medium and high clouds. In both cloud masks 1 represents overcast sky and 0 clear sky.

[3] Q.-Z. Ye and S.-S. Chen, The ultimate meteorological question from observational astronomers: how good is the cloud cover forecast?, Monthly Notices of the Royal Astronomical Society 428 (2013) 3288.

[4] A. Anzalone, M.E. Bertaina, S. Briz, C. Cassardo, R. Cremonini, A.J. de Castro et al., Methods to retrieve the cloud-top height in the frame of the JEM-EUSO mission, IEEE Transactions on geoscience and remote sensing 57 (2019) 304.

[5] P. Heidke, Berechnung des erfolges und der güte der windstärkevorhersagen im sturmwarnungsdienst, Geografiska Annaler 8 (1926) 301. 


\section{Full Authors List: JEM-EUSO Collaboration}

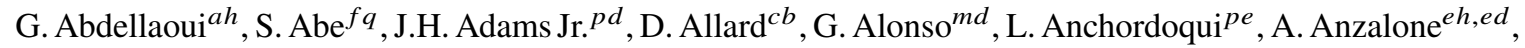
E. Arnone $e^{e k, e l}$, K. Asano ${ }^{f e}$, R. Attallah ${ }^{a c}$, H. Attoui ${ }^{a a}$, M. Ave Pernas ${ }^{m c}$, M. Bagheri ${ }^{p h}$, J. Baláz ${ }^{l a}$, M. Bakiri ${ }^{a a}$, D. Barghini ${ }^{e l, e k}$, S. Bartocci ${ }^{e i, e j}$, M. Battisti ${ }^{e k, e l}$, J. Bayer ${ }^{d d}$, B. Beldjilali ${ }^{a h}$, T. Belenguer ${ }^{m b}$, N. Belkhalfa ${ }^{a a}$, R. Bellotti ${ }^{e a, e b}$, A.A. Belov ${ }^{k b}$, K. Benmessai $^{a a}$, M. Bertaina $^{e k, e l}$, P.F. Bertone ${ }^{p f}$, P.L. Biermann ${ }^{d b}$, F. Bisconti ${ }^{e l, e k}$, C. Blaksley ${ }^{f t}$, N. Blanc ${ }^{o a}$, S. Blin-Bondil ${ }^{c a, c b}$, P. Bobik ${ }^{l a}$, M. Bogomilov ${ }^{b a}$, E. Bozzo ${ }^{o b}$, S. Briz ${ }^{p b}$, A. Bruno ${ }^{e h, e d}$, K.S. Caballero ${ }^{h d}$, F. Cafagna ${ }^{e a}$, G. Cambie ${ }^{e i, e j}$, D. Campana ${ }^{e f}$, J-N. Capdevielle $^{c b}$,

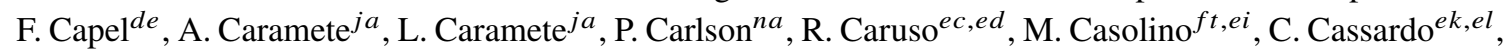

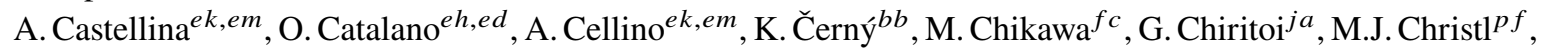
R. Colalillo ${ }^{e f, e g}$, L. Conti ${ }^{e n, e i}$, G. Cotto ${ }^{e k, e l}$, H.J. Crawford ${ }^{p a}$, R. Cremonini ${ }^{e l}$, A. Creusot ${ }^{c b}$, A. de Castro Gónzalez ${ }^{p b}$, C. de la Taille ${ }^{c a}$, L. del Peral ${ }^{m c}$, A. Diaz Damian ${ }^{c c}$, R. Diesing ${ }^{p b}$, P. Dinaucourt ${ }^{c a}$, A. Djakonow ${ }^{i a}$, T. Djemil ${ }^{a c}$, A. Ebersoldt ${ }^{d b}$, T. Ebisuzaki ${ }^{f t}$, L. Eliasson ${ }^{n a}$, J. Eser ${ }^{p b}$, F. Fenu $^{e k, e l}$, S. Fernández-González ${ }^{m a}$, S. Ferrarese ${ }^{e k, e l}$, G. Filippatos ${ }^{p c}$, W.I. Finch ${ }^{p c}$ C. Fornaro ${ }^{e n, e i}$, M. Fouka ${ }^{a b}$, A. Franceschi ${ }^{e e}$, S. Franchini ${ }^{m d}$, C. Fuglesang ${ }^{n a}$, T. Fujii ${ }^{f g}$, M. Fukushima ${ }^{f e}$, P. Galeotti ${ }^{e k, e l}$, E. GarcíaOrtega $^{m a}$, D. Gardiol ${ }^{e k, e m}$, G.K. Garipov ${ }^{k b}$, E. Gascón ${ }^{m a}$, E. Gazda ${ }^{p h}$, J. Genci ${ }^{l b}$, A. Golzio ${ }^{e k, e l}$, C. González Alvarado $^{m b}$, P. Gorodetzky ${ }^{f t}$, A. Green ${ }^{p c}$, F. Guarino ${ }^{e f, e g}$, C. Guépin ${ }^{p l}$, A. Guzmán ${ }^{d d}$, Y. Hachisu ${ }^{f t}$, A. Haungs ${ }^{d b}$, J. Hernández Carretero ${ }^{m c}$, L. Hulett ${ }^{p c}$, D. Ikeda ${ }^{f e}$, N. Inoue ${ }^{f n}$, S. Inoue ${ }^{f t}$, F. Isgrò ${ }^{e f, e g}$, Y. Itow ${ }^{f k}$, T. Jammer ${ }^{d c}$, S. Jeong ${ }^{g b}$, E. Joven ${ }^{m e}$, E.G. Judd ${ }^{p a}$, J. Jochum ${ }^{d c}$, F. Kajino $f f$, T. Kajino ${ }^{f i}$, S. Kalli ${ }^{a f}$, I. Kaneko ${ }^{f t}$, Y. Karadzhov ${ }^{b a}$, M. Kasztelan ${ }^{i a}$, K. Katahira ${ }^{f t}$, K. Kawai ${ }^{f t}$, Y. Kawasaki ${ }^{f t}$, A. Kedadra ${ }^{a a}$, H. Khales ${ }^{a a}$, B.A. Khrenov ${ }^{k b}$, Jeong-Sook Kim ${ }^{g a}$, Soon-Wook Kim ${ }^{g a}$, M. Kleifges ${ }^{d b}$, P.A. Klimov ${ }^{k b}$, D. Kolev ${ }^{b a}$, I. Kreykenbohm ${ }^{d a}$, J.F. Krizmanic ${ }^{p f, p k}$, K. Królik ${ }^{i a}$, V. Kungel ${ }^{p c}$, Y. Kurihara $^{f s}$, A. Kusenko ${ }^{f r, p e}$, E. Kuznetsov ${ }^{p d}$, H. Lahmar ${ }^{a a}$, F. Lakhdari ${ }^{a g}$, J. Licandro ${ }^{m e}$, L. López Campano ${ }^{m a}$, F. López Martínez ${ }^{p b}$, S. Mackovjak ${ }^{l a}$, M. Mahdi ${ }^{a a}$, D. Mandát ${ }^{b c}$, M. Manfrin $^{e k, e l}$, L. Marcelli $^{e i}$, J.L. Marcos $^{m a}$, W. Marszał ${ }^{i a}$, Y. Martín ${ }^{m e}$, O. Martinez ${ }^{h c}$, K. Mase ${ }^{f a}$, R. Matev ${ }^{b a}$, J.N. Matthews ${ }^{p g}$, N. Mebarki ${ }^{a d}$,

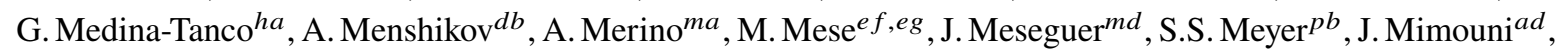
H. Miyamoto ${ }^{e k, e l}$, Y. Mizumoto ${ }^{f i}$, A. Monaco ${ }^{e a, e b}$, J.A. Morales de los Ríos ${ }^{m c}$, M. Mastafa $^{p d}$, S. Nagataki $^{f t}$,

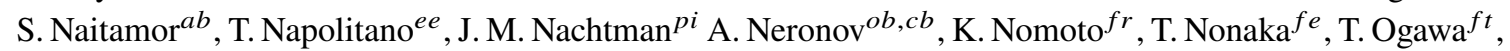

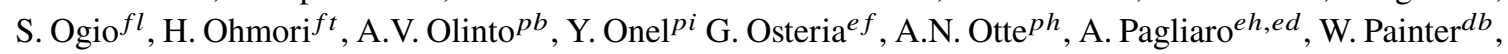
M.I. Panasyuk ${ }^{k b}$, B. Panico ${ }^{e f}$, E. Parizot ${ }^{c b}$, I.H. Park ${ }^{g b}$, B. Pastircak ${ }^{l a}$, T. Paul ${ }^{p e}$, M. Pech ${ }^{b b}$, I. Pérez-

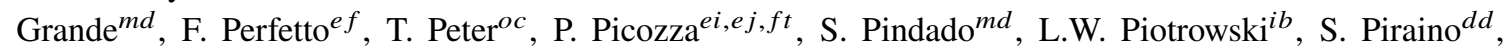
Z. Plebaniak ${ }^{e k, e l, i a}$, A. Pollini ${ }^{o a}$, E.M. Popescu ${ }^{j a}$, R. Prevete $^{e f, e g}$, G. Prévôt $^{c b}$, H. Prieto $^{m c}$, M. Przybylak $^{i a}$, G. Puehlhofer ${ }^{d d}$, M. Putis ${ }^{l a}$, P. Reardon ${ }^{p d}$, M.H.. Reno ${ }^{p i}$, M. Reyes ${ }^{m e}$, M. Ricciee ${ }^{e}$ M.D. Rodríguez Frías ${ }^{m c}$, O.F. Romero Matamala ${ }^{p h}$, F. Ronga ${ }^{e e}$, M.D. Sabau ${ }^{m b}$, G. Saccáec,ed, G. Sáez Cano ${ }^{m c}$, H. Sagawa ${ }^{f e}$, Z. Sahnoune ${ }^{a b}$, A. Saito ${ }^{f g}$, N. Sakaki ${ }^{f t}$, H. Salazar ${ }^{h c}$, J.C. Sanchez Balanzar ${ }^{h a}$, J.L. Sánchez ${ }^{m a}$, A. Santangelo ${ }^{d d}$, A. Sanz-Andrés ${ }^{m d}$, M. Sanz Palomino ${ }^{m b}$, O.A. Saprykin ${ }^{k c}$, F. Sarazin ${ }^{p c}$, M. Sato ${ }^{f o}$, A. Scagliola ${ }^{e a, e b}$, T. Schanz ${ }^{d d}$, H. Schieler ${ }^{d b}$, P. Schovánek ${ }^{b c}$, V. Scotti ${ }^{e f, e g}$, M. Serra ${ }^{m e}$, S.A. Sharakin ${ }^{k b}$, H.M. Shimizu ${ }^{f j}$, K. Shinozaki ${ }^{i a}$, T. Shirahama ${ }^{f n}$, J.F. Soriano ${ }^{p e}$, A. Sotgiu ${ }^{e i, e j}$, I. Stan ${ }^{j a}$, I. Strharskýla ${ }^{l a}$ N. Sugiyama ${ }^{f j}$, D. Supanitsky ${ }^{h a}$, M. Suzuki ${ }^{f m}$, J. Szabelski ${ }^{i a}$, N. Tajima ${ }^{f t}$, T. Tajima ${ }^{f t}$, Y. Takahashi ${ }^{f o}$, M. Takeda ${ }^{f e}$, Y. Takizawa ${ }^{f t}$, M.C. Talai ${ }^{a c}$, Y. Tameda ${ }^{f u}$, C. Tenzer ${ }^{d d}$, S.B. Thomas ${ }^{p g}$, O. Tibolla ${ }^{h e}$, L.G. Tkachev ${ }^{k a}$, T. Tomida ${ }^{f h}$, N. Tone ${ }^{f t}$, S. Toscano ${ }^{o b}$, M. Traïche ${ }^{a a}$, Y. Tsunesada ${ }^{f l}$, K. Tsuno ${ }^{f t}$, S. Turriziani ${ }^{f t}$, Y. Uchihori ${ }^{f b}$, O. Vaduvescu ${ }^{m e}$, J.F. Valdés-Galicia ${ }^{h a}$, P. Vallania ${ }^{e k, e m}$, L. Valore ${ }^{e f, e g}$, G. VankovaKirilova $^{b a}$, T. M. Venters ${ }^{p j}$, C. Vigorito ${ }^{e k, e l}$, L. Villaseñor ${ }^{h b}$, B. Vlcek ${ }^{m c}$, P. von Ballmoos ${ }^{c c}$, M. Vrabel ${ }^{l b}$, S. Wada ${ }^{f t}$, J. Watanabe ${ }^{f i}$, J. Watts Jr. ${ }^{p d}$, R. Weigand Muñoz ${ }^{m a}$, A. Weindl ${ }^{d b}$ L. Wiencke $^{p c}$, M. Wille ${ }^{d a}$, J. Wilms ${ }^{d a}$, D. Winn ${ }^{p m}$ T. Yamamoto ${ }^{f f}$, J. Yang ${ }^{g b}$, H. Yano ${ }^{f m}$, I.V. Yashin ${ }^{k b}$, D. Yonetoku ${ }^{f d}$, S. Yoshida $^{f a}$, R. Young ${ }^{p f}$, I.S Zgura ${ }^{j a}$, M.Yu. Zotov ${ }^{k b}$, A. Zuccaro Marchi ${ }^{f t}$

aa Centre for Development of Advanced Technologies (CDTA), Algiers, Algeria

$a b$ Dep. Astronomy, Centre Res. Astronomy, Astrophysics and Geophysics (CRAAG), Algiers, Algeria 
${ }^{a c}$ LPR at Dept. of Physics, Faculty of Sciences, University Badji Mokhtar, Annaba, Algeria

${ }^{a d}$ Lab. of Math. and Sub-Atomic Phys. (LPMPS), Univ. Constantine I, Constantine, Algeria

af Department of Physics, Faculty of Sciences, University of M'sila, M'sila, Algeria

ag Research Unit on Optics and Photonics, UROP-CDTA, Sétif, Algeria

${ }^{a h}$ Telecom Lab., Faculty of Technology, University Abou Bekr Belkaid, Tlemcen, Algeria

ba St. Kliment Ohridski University of Sofia, Bulgaria

$b b$ Joint Laboratory of Optics, Faculty of Science, Palacký University, Olomouc, Czech Republic

${ }^{b c}$ Institute of Physics of the Czech Academy of Sciences, Prague, Czech Republic

ca Omega, Ecole Polytechnique, CNRS/IN2P3, Palaiseau, France

${ }^{c b}$ Université de Paris, CNRS, AstroParticule et Cosmologie, F-75013 Paris, France

${ }^{c c}$ IRAP, Université de Toulouse, CNRS, Toulouse, France

$d a$ ECAP, University of Erlangen-Nuremberg, Germany

${ }^{d b}$ Karlsruhe Institute of Technology (KIT), Germany

$d c$ Experimental Physics Institute, Kepler Center, University of Tübingen, Germany

$d d$ Institute for Astronomy and Astrophysics, Kepler Center, University of Tübingen, Germany

de Technical University of Munich, Munich, Germany

ea Istituto Nazionale di Fisica Nucleare - Sezione di Bari, Italy

${ }^{e b}$ Universita' degli Studi di Bari Aldo Moro and INFN - Sezione di Bari, Italy

ec Dipartimento di Fisica e Astronomia "Ettore Majorana", Universitá di Catania, Italy

${ }^{e d}$ Istituto Nazionale di Fisica Nucleare - Sezione di Catania, Italy

$e e$ Istituto Nazionale di Fisica Nucleare - Laboratori Nazionali di Frascati, Italy

ef Istituto Nazionale di Fisica Nucleare - Sezione di Napoli, Italy

eg UniversitaÕ di Napoli Federico II - Dipartimento di Fisica "Ettore Pancini", Italy

eh INAF - Istituto di Astrofisica Spaziale e Fisica Cosmica di Palermo, Italy

${ }^{e i}$ Istituto Nazionale di Fisica Nucleare - Sezione di Roma Tor Vergata, Italy

${ }^{e j}$ Universita' di Roma Tor Vergata - Dipartimento di Fisica, Roma, Italy

${ }^{e k}$ Istituto Nazionale di Fisica Nucleare - Sezione di Torino, Italy

el Dipartimento di Fisica, Universita' di Torino, Italy

em Osservatorio Astrofisico di Torino, Istituto Nazionale di Astrofisica, Italy

en Uninettuno University, Rome, Italy

fa Chiba University, Chiba, Japan

${ }^{f b}$ National Institutes for Quantum and Radiological Science and Technology (QST), Chiba, Japan

$f c$ Kindai University, Higashi-Osaka, Japan

$f d$ Kanazawa University, Kanazawa, Japan

$f e$ Institute for Cosmic Ray Research, University of Tokyo, Kashiwa, Japan

ff Konan University, Kobe, Japan

$f g$ Kyoto University, Kyoto, Japan

$f^{h}$ Shinshu University, Nagano, Japan

${ }^{f i}$ National Astronomical Observatory, Mitaka, Japan

$f j$ Nagoya University, Nagoya, Japan

${ }^{f k}$ Institute for Space-Earth Environmental Research, Nagoya University, Nagoya, Japan

${ }^{f l}$ Graduate School of Science, Osaka City University, Japan

${ }^{f m}$ Institute of Space and Astronautical Science/JAXA, Sagamihara, Japan

${ }^{f n}$ Saitama University, Saitama, Japan

fo Hokkaido University, Sapporo, Japan

$f p$ Osaka Electro-Communication University, Neyagawa, Japan

${ }^{f q}$ Nihon University Chiyoda, Tokyo, Japan

$f r$ University of Tokyo, Tokyo, Japan 
$f s$ High Energy Accelerator Research Organization (KEK), Tsukuba, Japan

${ }^{f t}$ RIKEN, Wako, Japan

ga Korea Astronomy and Space Science Institute (KASI), Daejeon, Republic of Korea

$g b$ Sungkyunkwan University, Seoul, Republic of Korea

ha Universidad Nacional Autónoma de México (UNAM), Mexico

$h b$ Universidad Michoacana de San Nicolas de Hidalgo (UMSNH), Morelia, Mexico

$h c$ Benemérita Universidad Autónoma de Puebla (BUAP), Mexico

${ }^{h d}$ Universidad Autónoma de Chiapas (UNACH), Chiapas, Mexico

he Centro Mesoamericano de Física Teórica (MCTP), Mexico

ia National Centre for Nuclear Research, Lodz, Poland

${ }^{i b}$ Faculty of Physics, University of Warsaw, Poland

${ }^{j a}$ Institute of Space Science ISS, Magurele, Romania

$k a$ Joint Institute for Nuclear Research, Dubna, Russia

${ }^{k b}$ Skobeltsyn Institute of Nuclear Physics, Lomonosov Moscow State University, Russia

$k c$ Space Regatta Consortium, Korolev, Russia

la Institute of Experimental Physics, Kosice, Slovakia

$l b$ Technical University Kosice (TUKE), Kosice, Slovakia

$m a$ Universidad de León (ULE), León, Spain

$m b$ Instituto Nacional de Técnica Aeroespacial (INTA), Madrid, Spain

${ }^{m c}$ Universidad de Alcalá (UAH), Madrid, Spain

$m d$ Universidad Politécnia de madrid (UPM), Madrid, Spain

me Instituto de Astrofísica de Canarias (IAC), Tenerife, Spain

${ }^{n a}$ KTH Royal Institute of Technology, Stockholm, Sweden

oa Swiss Center for Electronics and Microtechnology (CSEM), Neuchâtel, Switzerland

$o b$ ISDC Data Centre for Astrophysics, Versoix, Switzerland

${ }^{o c}$ Institute for Atmospheric and Climate Science, ETH Zürich, Switzerland

$p a$ Space Science Laboratory, University of California, Berkeley, CA, USA

${ }^{p b}$ University of Chicago, IL, USA

${ }^{p c}$ Colorado School of Mines, Golden, CO, USA

${ }^{p d}$ University of Alabama in Huntsville, Huntsville, AL; USA

$p e$ Lehman College, City University of New York (CUNY), NY, USA

$p f$ NASA Marshall Space Flight Center, Huntsville, AL, USA

$p g$ University of Utah, Salt Lake City, UT, USA

${ }^{p h}$ Georgia Institute of Technology, USA

${ }^{p i}$ University of Iowa, Iowa City, IA, USA

$p j$ NASA Goddard Space Flight Center, Greenbelt, MD, USA

${ }^{p k}$ Center for Space Science \& Technology, University of Maryland, Baltimore County, Baltimore, MD, USA

${ }^{p l}$ Department of Astronomy, University of Maryland, College Park, MD, USA

${ }^{p m}$ Fairfield University, Fairfield, CT, USA 